\title{
Electron coherent states in strained graphene
}

\section{E. Díaz-Bautista ${ }^{a}$, Y. Concha-Sánchez ${ }^{* b}$ and A. Raya ${ }^{c}$}

${ }^{a}$ Departamento de Formación Básica Disciplinaria, Unidad Profesional Interdisciplinaria de Ingeniería Campus Hidalgo del Instituto Politécnico Nacional, Pachuca: Ciudad del Conocimiento y la Cultura, Carretera Pachuca-Actopan km 1+500, San Agustín Tlaxiaca, 42162 Hidalgo, Mexico

${ }^{b}$ Facultad de Ingeniería Civil, Universidad Michoacana de San Nicolás de Hidalgo, Edificio C, Ciudad Universitaria. Francisco J. Mújica s/n. Col. Felícitas del Río. 58030, Morelia, Michoacán, México

${ }^{c}$ Instituto de Física y Matemáticas, Universidad Michoacana de San Nicolás de Hidalgo, Edificio C-3, Ciudad Universitaria. Francisco J. Mújica s/n. Col. Felícitas del Río. 58040 Morelia, Michoacán, México

E-mail: ediazba@ipn.mx, yconcha@umich.mx, raya@ifm.umich.mx

\begin{abstract}
We construct electron coherent states in strained graphene immersed in a constant homogeneous magnetic field which is orthogonal to the sample surface. We consider the situation in which the membrane is deformed uniformily and uniaxially, avoiding the generation of pseudo-magnetic fields and solve the Dirac-Weyl equation with an anisotropic Fermi velocity, identifying the appropriate rising and lowering operators. Working in a Landau gauge, we explicitly construct nonlinear coherent states as eigenstates of a generalized annihilation operator with complex eigenvalues which depends on an arbitrary function $f$ of the number operator. In order to describe the anisotropy effects on these states, we obtain density probability for three different functions $f$.
\end{abstract}

European Physical Society Conference on High Energy Physics - EPS-HEP2019 -

10-17 July, 2019

Ghent, Belgium

\footnotetext{
${ }^{*}$ Speaker.
} 

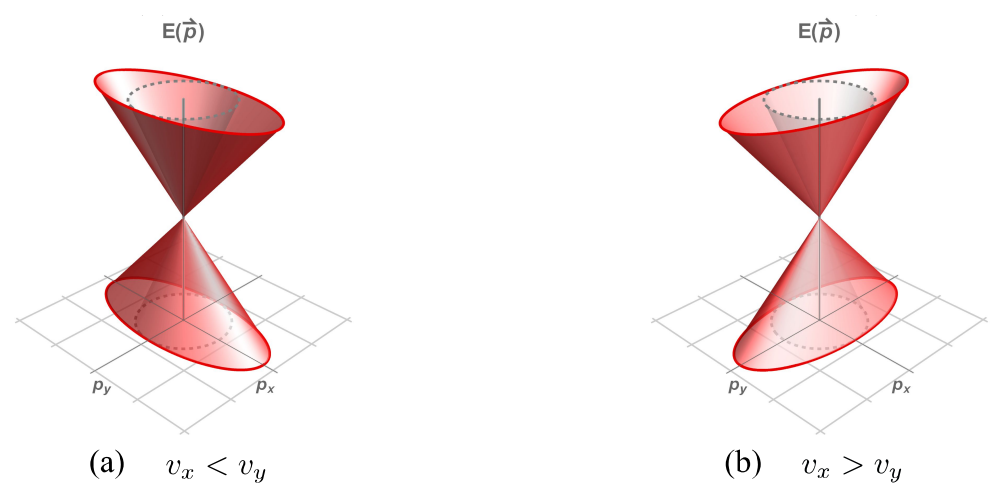

Figure 1: Dirac cones for pristine (dashed gray contour) and uniaxially strained graphene (solid red contour). For former, the Dirac cones projections on the horizontal plane are circles, while for latter, such projections are ellipses whose semi-major axis is along either (a) of the $p_{x}$-axis when $v_{x}<v_{y}$ or (b) of the $p_{y}$-axis when $v_{x}>v_{y}$. Being precise, when strain is applied the Dirac cones are displaced out from their original positions.

\section{Introduction}

The physical system of a graphene layer interacting with a uniform magnetic field has been considered in several works due to its important technological implications [1, 2, 3, 4]. This material is characterized because, at the continuum limit, the behavior of its charge carriers (generally referred to as electrons) mimics that of ultra-relativistic fermions. Thus, its dispersion relation is linear and the quasiparticles are described by a Dirac-like equation. Several phenomena related to such pseudo-relativistic behavior have been studied extensively, including when a graphene sample is mechanical deformed. Therefore, our main interest is to provide a semi-classical description of the strain effects on electrons in graphene by employing nonlinear coherent states (NLCSs), which are defined through a nonlinear or $f$-deformed algebra.

\section{Dirac-Weyl equation under strain}

Let us start from the anisotropic Dirac-Weyl equation under a homogeneous magnetic field

$$
H_{D} \Psi(x, y)=\left(v_{x} \sigma_{x} \pi_{x}+v_{y} \sigma_{y} \pi_{y}\right) \Psi(x, y)=E \Psi(x, y),
$$

where $v_{x}=a v_{\mathrm{F}}$ and $v_{y}=b v_{\mathrm{F}}$, with $a$ and $b$ being positive real numbers, are the electron velocities in the directions $x$ and $y$ when a uniform uniaxial strain is applied, and $v_{\mathrm{F}}$ is the Fermi velocity (see Fig. 1). Here $\Psi(x, y)=\left(\psi^{+}(x, y) \psi^{-}(x, y)\right)^{\mathrm{T}}$ and $\pi_{x, y}=p_{x, y}+e A_{x, y}$, where $\vec{p}$ denotes the canonical momentum while $\vec{A}$ is the vector potential that defines a magnetic field aligned perpendicularly to the graphene surface. In a Landau gauge, we set $\vec{A}=B_{0} x \hat{j}$ and $\vec{B}=\nabla \times \vec{A}=B_{0} \hat{k}$.

Substituting the above expressions in Eq. (2.1), we get two decoupled equations, namely,

$$
\left[-\frac{d^{2}}{d x^{2}}+\frac{\omega_{\zeta}^{2}}{4}\left(x+\frac{2 k}{\omega}\right)^{2} \pm \frac{1}{2} \omega_{\zeta}\right] \psi^{ \pm}(x)=\varepsilon_{a}^{ \pm 2} \psi^{ \pm}(x),
$$

where $\varepsilon_{a}^{ \pm}=E /\left(a v_{\mathrm{F}} \hbar\right), \zeta=v_{x} / v_{y}=a / b$ and the frequency $\omega_{\zeta}=\omega / \zeta=2 e B_{0} /(\zeta \hbar)$, being $\omega$ the cyclotron frequency of electrons in pristine graphene. 
After solving the ODEs in (2.2), it follows that:

$$
E_{0}^{-}=0, \quad E_{n}^{-}=E_{n-1}^{+}= \pm v_{\mathrm{F}} \hbar \sqrt{a b \omega n}, \quad n=0,1,2, \ldots
$$

where positive (negative) energies correspond to the conduction (valence) band, while the pseudospinor eigenstates turn out to be

$$
\Psi_{n}(x, y)=\frac{\exp (i k y)}{\sqrt{2^{\left(1-\delta_{0 n}\right)}}}\left(\begin{array}{c}
\left(1-\delta_{0 n}\right) \psi_{n-1}(x) \\
i \psi_{n}(x)
\end{array}\right), \quad n=0,1,2, \ldots,
$$

where $\delta_{m n}$ denotes the Kronecker delta, $\psi_{n}^{-} \equiv \psi_{n}$ and $\psi_{n}^{+} \equiv \psi_{n-1}$ are eigenfunctions of the shifted harmonic oscillator (2.2).

\section{Annhilation operator}

In order to build nonlinear coherent states in strained graphene, one can define a deformed annihilation operator $\Theta_{f}$ given by [5]:

$$
\Theta_{f}^{-}=\left[\begin{array}{cc}
\cos (\delta) \frac{\sqrt{N+2}}{\sqrt{N+1}} f(N+2) \theta^{-} & \sin (\delta) \frac{f(N+2)}{\sqrt{N+1}}\left(\theta^{-}\right)^{2} \\
-\sin (\delta) f(N+1) \sqrt{N+1} & \cos (\delta) f(N+1) \theta^{-}
\end{array}\right], \quad \theta^{ \pm}=\frac{1}{\sqrt{2}}\left(\mp \frac{d}{d \xi}+\xi\right),
$$

where $\theta^{+}=\left(\theta^{-}\right)^{\dagger}$ and $\xi=\sqrt{\omega_{\zeta} / 2}(x+2 k / \omega)$. The action of $\Theta_{f}^{-}$on the eigenstates (2.4) is

$$
\Theta_{f}^{-} \Psi_{n}(x, y)=2^{-\delta_{1 n} / 2} f(n) \exp (i \delta) \sqrt{n} \Psi_{n-1}(x, y), \quad n=0,1,2, \ldots,
$$

being $f(N)$ a well-behaved function of the number operator $N=\theta^{+} \theta^{-}$and $\delta \in[0,2 \pi]$ allows to consider either diagonal or non-diagonal matrix representation for $\Theta_{f}^{-}$.

Taking into account the generalized creation operator $\Theta_{f}^{+}=\left(\Theta_{f}^{-}\right)^{\dagger}$, we obtain the nonlinear algebra $\left[\Theta_{f}^{-}, \Theta_{f}^{+}\right]=\operatorname{diag}(\Omega(N+1), \Omega(N))$, where $\Omega(N)=(N+1) f^{2}(N+1)-N f^{2}(N)$. In the limit $f(N)=1$ we recover the Heisenberg-Weyl algebra, namely, $\left[\Theta_{f}^{-}, \Theta_{f}^{+}\right]=\mathbb{I}$, where $\mathbb{I}$ is the $2 \times 2$ unity matrix.

\subsection{Nonlinear coherent states}

We can construct NLCSs $\Psi_{\alpha}^{f}(x, y)$ as eigenstates of the operator $\Theta_{f}^{-}$with eigenvalue $\alpha \in \mathbb{C}$ :

$$
\Theta_{f}^{-} \Psi_{\alpha}^{f}(x, y)=\alpha \Psi_{\alpha}^{f}(x, y), \quad \text { where } \quad \Psi_{\alpha}^{f}(x, y)=a_{0} \Psi_{0}(x, y)+\sum_{n=1}^{\infty} a_{n} \Psi_{n}(x, y) .
$$

Upon inserting these states into the corresponding eigenvalue equation, we get the following relations:

$$
a_{1} f(1)=\sqrt{2} \tilde{\alpha} a_{0}, \quad a_{n+1} f(n+1) \sqrt{n+1}=\tilde{\alpha} a_{n},
$$

with $\tilde{\alpha}=\alpha \exp (-i \delta)$. Then [5]:

$$
\Psi_{\alpha}^{f}(x, y)=\left\{\begin{array}{lr}
\frac{1}{\sqrt{2 \exp \left(|\tilde{\alpha}|^{2}\right)-1}}\left[\Psi_{0}(x, y)+\sum_{n=1}^{\infty} \frac{\sqrt{2} \tilde{\alpha}^{n}}{\sqrt{n !}} \Psi_{n}(x, y)\right], & \text { if } f(N+1)=1, \\
\exp \left(-|\tilde{\alpha}|^{2} / 2\right) \sum_{n=0}^{\infty} \frac{\tilde{\alpha}^{n}}{n !} \Psi_{n+1}(x, y), & \text { if } \quad f(N+1)=\frac{\sqrt{N}}{\sqrt{N+\hat{1}}}, \\
\left(\frac{|\tilde{\alpha}|}{I_{1}(2|\tilde{\alpha}|)}\right)^{1 / 2} \sum_{n=0}^{\infty} \frac{\tilde{\alpha}^{n}}{\sqrt{n !(n+1) !}} \Psi_{n+2}(x, y), & \text { if } \quad f(N+2)=\frac{\sqrt{N} \sqrt{N+\hat{1}}}{\sqrt{N+\hat{2}}} .
\end{array}\right.
$$

whose probability densities are depicted in the Figure 2. 
(a)

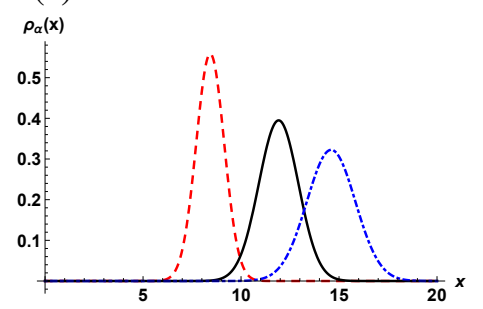

(b)

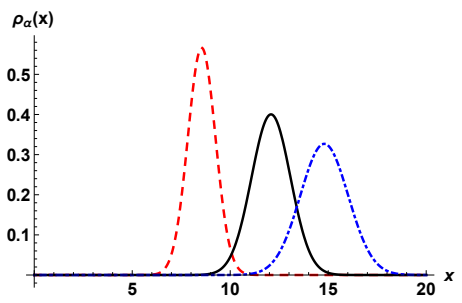

(c)

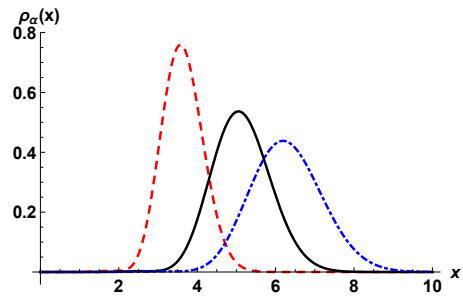

Figure 2: Probability density $\rho_{\alpha}(x)=\left|\Psi_{\alpha}^{f}(x, y)\right|^{2}$ for each NLCS in Eq. (3.5) with real eigenvalue $\alpha=6$ : (a) $f(N+1)=1$, (b) $f(N+1)=\sqrt{N} / \sqrt{N+1}$ and (c) $f(N+2)=\sqrt{N} \sqrt{N+1} / \sqrt{N+2}$. The pristine graphene case is identified by $\zeta=1$ (-), whereas the deformed graphene case is labeled by $\zeta=1 / 2(---)$, when strain is applied along the $x$ - (or zig-zag) direction, and $\zeta=3 / 2(-\cdots-)$, when strain is applied along the $y$ (or arm-chair) direction. In all these cases, we take $B_{0}=1 / 2, \omega=1$ and $k=\delta=0$.

\section{Conclusions}

In this work, we have considered a uniform and uniaxially strained graphene membrane immersed in a perpendicular uniform magnetic field, in order to explore the mechanical deformation effects have in the behavior of the nonlinear coherent states for electrons, which have been obtained by describing the background field in a Landau gauge. Thus, if $\zeta<1\left(v_{x}<v_{y}\right)$, the deformation takes place along the $x$-direction and the velocity $v_{x}$ decreases. Therefore, as a consequence of the Heisenberg uncertainty principle, the probability density of the NLCS is larger in comparison with the opposite case, $\zeta>1\left(v_{x}>v_{y}\right)$, in which the strain is applied along the $y$-direction and the velocity $v_{y}$ decreases (see Fig. 2). On the other hand, the function $\rho_{\alpha}(x)$ of the third family of NLCSs seems not to be affected by strain in the same way as the other coherent states as a result, we think, of the function $f$ that defines it. In [5], the occupation number distribution $P_{\alpha}(n)=\left|\left\langle\Psi_{n} \mid \Psi_{\alpha}^{f}\right\rangle\right|^{2}$ is discussed to analyze this behavior.

It is worth to mention that this physical problem has been generalized and addressed by defining the external field in the symmetric gauge [6], in order to describe the bidimensional effects of anisotropy in strained graphene and 2D Dirac materials in general.

\section{References}

[1] K. S. Novoselov, E. McCann, S. V Morozov, V. I. Falko, M.I. Katsnelson, U. Zeitler, D. Jiang, F. Schedin, A. K. Geim, Nat. Phys. 2177 (2006).

[2] M.I. Katsnelson, Mater. Today 1020 (2007).

[3] K. S. Novoselov, Z. Jiang, Y. Zhang, S. V. Morozov, H. L. Stormer, U. Zeitler, J. C. Maan, G. S. Boe-binger, P. Kim, A. K. Geim, Science 3151379 (2007).

[4] A. K. Geim, K. S. Novoselov, Nat. Mater. 6183 (2007).

[5] E. Díaz-Bautista, Y. Concha-Sánchez, A. Raya, J. Phys.: Condens. Matter 31435702 (2019).

[6] E. Díaz-Bautista, M. Oliva-Leyva, Y. Concha-Sánchez, A. Raya, Coherent states in magnetized anisotropic 2D-Dirac materials (2019) [quant-ph/1907.06551]. 